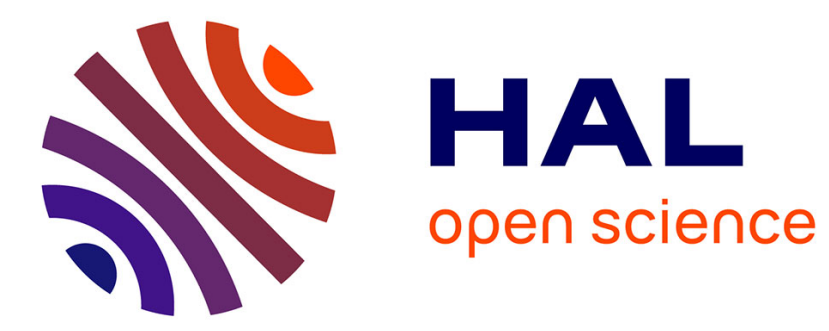

\title{
On the use of leaky modes in open waveguides for the sound propagation modeling in street canyons
}

\author{
Adrien Pelat, Simon Félix, Vincent Pagneux
}

\section{To cite this version:}

Adrien Pelat, Simon Félix, Vincent Pagneux. On the use of leaky modes in open waveguides for the sound propagation modeling in street canyons. Journal of the Acoustical Society of America, 2009, 126 (6), pp.2864-2872. 10.1121/1.3259845 . hal-02453272

\section{HAL Id: hal-02453272 \\ https://hal-univ-lemans.archives-ouvertes.fr/hal-02453272}

Submitted on 15 Dec 2020

HAL is a multi-disciplinary open access archive for the deposit and dissemination of scientific research documents, whether they are published or not. The documents may come from teaching and research institutions in France or abroad, or from public or private research centers.
L'archive ouverte pluridisciplinaire HAL, est destinée au dépôt et à la diffusion de documents scientifiques de niveau recherche, publiés ou non, émanant des établissements d'enseignement et de recherche français ou étrangers, des laboratoires publics ou privés. 


\title{
On the use of leaky modes for the sound propagation modeling in an open urban street canyon
}

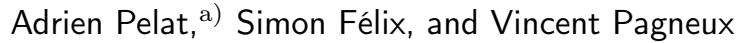 \\ LAUM, CNRS, Université du Maine, avenue Olivier Messiaen, 72085 Le Mans, France.
}

\begin{abstract}
One is interested in a multimodal approach to describe the sound propagation within an urban, U-shaped, street canyon being considered as an open waveguide in which the sound may propagate. The key point in such a multimodal formalism is the choice of the basis of local transversal modes on which the acoustic field is decomposed. For a waveguide with a simple and bounded cross-section, a complete orthogonal basis can be analytically obtained. The case of an open waveguide is more difficult, since no such a basis can be exhibited. However, an open resonator, as for example the U-shaped cross-section of a street, presents resonant modes with complex eigenfrequencies, owing to radiative losses. This work first presents how to numerically obtain these modes. Results for the transverse modes are also compared with solutions obtained by the Finite Elements Method (FEM) with Perfectly Matched Layers (PML). Then, examples are treated to show how these leaky modes can be used as a basis for the modal decomposition of the sound field in a street canyon.
\end{abstract}

PACS numbers: $43.20 . \mathrm{Mv}, 43.28 . \mathrm{Js}, 43.50 . \mathrm{Vt}$

\section{INTRODUCTION}

The aim of the present work is the resolution of the wave equation

$$
\left(\vec{\nabla}^{2}-\frac{1}{c_{0}^{2}} \frac{\partial^{2}}{\partial t^{2}}\right) p=0
$$

in a long open rectangular enclosure (Fig. 1), to model the sound propagation within a street canyon.

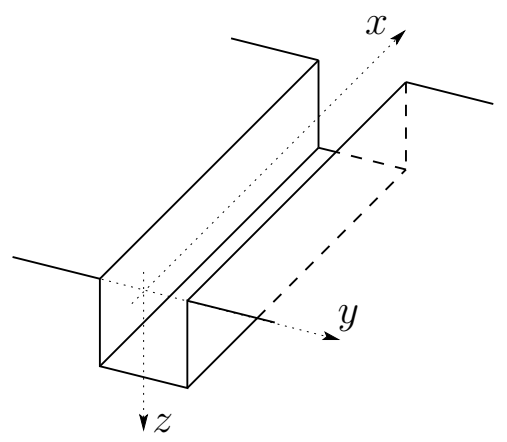

Figure 1. A straight open waveguide as model of a street canyon.

The investigation of sound propagation in urban environments and streets has been the subject of extensive researches in the past four decades, as the response to a growing social demand.

After experimental observations? ? ? in the 60's, the earliest theoretical works on this topic were conducted in the 70's. Davies? , Lee? , Stenackers et al. ? , and Lyon? used image sources to study multiple sound reflections in a street considered as a channel between two infinite

\footnotetext{
a) Electronic address: adrien.pelat.etu@univ-lemans.fr
}

walls. Later, image source method has been improved considering scattering at façade irregularities? ${ }^{\text {, diffusely }}$ reflecting façades? ${ }^{\text {or coherent image sources? }}$. Other energetic approach were also used in urban acoustics. Kang? developped a radiosity based model, Bradley? used ray tracing method, Picaut et al. ${ }^{\text {? }}$ proposed a method based on a diffusion equation governing sound particles propagation. These energetic approaches give statistical description of sound fields in urban environment and are able to model more or less accurately numerous phenomena occcuring in streets. Since these approaches assume high frequency hypothesis, they cannot describe sound fields when the wavelength is in the range of street width. Furthermore, computation costs strongly increase for complex geometries or for 3D problems.

As pointed out by some authors? ? , interference effects are significant for relatively narrow street canyons (i.e. when the wavelength of the audible sound is of the same order of magnitude as the street width). Hence, wave methods present real interests for sound propagation modeling. Bullen and Fricke? ?, thirty years ago studied the wave propagation in streets using an approximated 2D modal approach, notably to model junctions of streets. To solve the wave equation in a 2D street, Finite Elements or Boundary Elements methods? could as well give solutions to the wave equation for realistic geometries, but the high computation costs restrict their use to low frequencies or to $2 \mathrm{D}$ approximations. The equivalent sources approach? ? ? is field-based rather than raybased, and apprehends the resonant behaviour of a city canyon. In this approach, a set of equivalent sources are used to couple the free half space above the canyon to the cavity inside the canyon. The finite differences in time domain (FDTD) method describes the sound field in $2 \mathrm{D}$ or even $3 \mathrm{D}$ problems? ${ }^{\text {? }}$ and can model a priori a very large number of phenomena. The parabolic equation coupled with FDTD method can also be useful to take into account meteorological effects? ${ }^{\text {}}$. These wave methods, however, are based on strong approximations or, for fully numerical methods, it lacks an explicit physical in- 
terpretation of the relationship between the properties of the sound field and, e.g., the geometry of the street.

The aim of the present paper is to give a multimodal description of the wave propagation in a 3D street canyon, this latter being regarded as a straight open waveguide. In a modal approach within a uniform waveguide, elementary solutions for the pressure are written as

$$
p_{i}(x, y, z)=e^{\mathrm{j}\left( \pm k_{i} x-\omega t\right)} \phi_{i}(y, z)
$$

where $k_{i}^{2}=k^{2}-\alpha_{i}^{2}, \Re\left\{k_{i}\right\}>0, \Im\left\{k_{i}\right\}>0, k=\omega / c_{0}$, and $\left(\alpha_{i}, \phi_{i}\right)$ are the eigensolutions of the transverse eigenproblem

$$
\vec{\nabla}_{\perp}^{2} \phi=-\alpha^{2} \phi
$$

with proper boundary conditions, in the cross-section of the waveguide. Then, a solution in the waveguide can be built as a sum on these elementary solutions (the time dependence $\exp (-\mathrm{j} \omega t)$ is omitted):

$$
p(x, y, z, \omega)=\sum_{i \in \mathbb{N}^{*}}\left(a_{i} e^{\mathrm{j} k_{i} x}+b_{i} e^{-\mathrm{j} k_{i} x}\right) \phi_{i}(y, z),
$$

and the coefficients $a_{i}$ and $b_{i}$ are found as functions of the end conditions in the waveguide.

In the classical case of closed waveguides having simple and bounded cross-section, a complete orthogonal modal basis $\left\{\phi_{i}\right\}$ can be analytically obtained. The case of open waveguides, as the one shown in Fig. 1, with a partially bounded cross-section, is more difficult, since no such a basis can be exhibited. However, a cross-section being regarded as an open resonator also displays eigenmodes with complex eigenfrequencies, owing to the radiation losses. ? ? ? ?

In this paper we propose to describe how the resonant modes of the open cross-section of an open waveguide (Fig. 1) can be used to give a multimodal formulation of the sound propagation in long open enclosures. A general method to compute the resonant frequencies and mode shapes in the transversal open cross-section of the duct is described. Results of the transverse problem are also compared with FEM computations using PML. Then, the multimodal propagation in a straight open waveguide is formulated and numerical examples are given and discussed.

\section{EIGENMODES OF THE TRANSVERSE PROBLEM}

\section{A. Theory and formulation}

The transverse modes of the open waveguide shown in Fig. 1 are written as the solutions $(\alpha, \phi)$ of the eigenproblem (3) with boundary conditions

$$
\begin{array}{ll}
\partial_{z} \phi=0 & \text { if } z=0 \text { and }|y|>L / 2, \\
\partial_{z} \phi=0 & \text { if } z=d \text { and }|y|<L / 2, \\
\partial_{y} \phi=0 & \text { if } y= \pm L / 2 \text { and } 0<z<d
\end{array}
$$

in the cross-section of the waveguide, regarded as a $2 \mathrm{D}$ rectangular cavity $\Omega_{1}$ open on the semi-infinite space
$\Omega_{2}$. Moreover, $\phi$ is an outgoing wave at infinity. A similar problem, with elastic waves, has been treated by Maradudin and Ryan? and part of the following equations are derived from this work (Fig. 2).

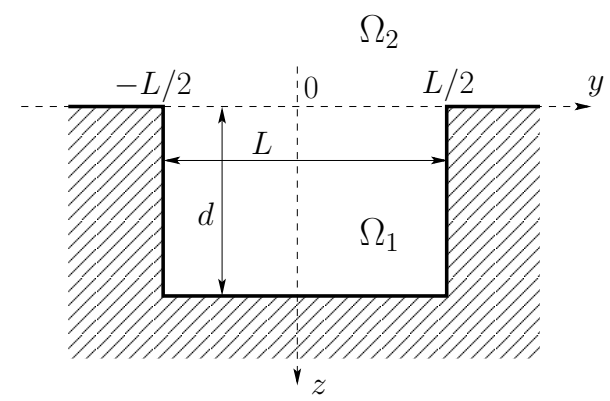

Figure 2. The cross-section of a street canyon seen as a $2 \mathrm{D}$ open rectangular cavity.

In the cavity $(z \geq 0)$, the general solution satisfying boundary conditions can be written as a discrete sum of functions,

$$
\phi(y, z \geq 0)=\sum_{n \in \mathbb{N}} A_{n} \cos \left(\beta_{n}(z-d)\right) \psi_{n}(y)
$$

with $d$ the depth of the cavity, $\beta_{n}^{2}=\alpha^{2}-\left(\frac{n \pi}{L}\right)^{2}$ and

$$
\psi_{n}(y)=\sqrt{2-\delta_{n 0}} \cos \left(\frac{n \pi}{L}\left(y-\frac{L}{2}\right)\right),
$$

where $\delta_{m n}$ is the Kronecker symbol.

Above the cavity $(z \leq 0)$, the general solution is written as the spatial Fourier transform

$$
\phi(y, z \leq 0)=\frac{1}{2 \pi} \int_{-\infty}^{+\infty} B e^{\mathrm{j}\left(\alpha_{y} y-\alpha_{z} z\right)} \mathrm{d} \alpha_{y},
$$

where $\alpha_{z}^{2}=\alpha^{2}-\alpha_{y}^{2}$ with $\Re\left\{\alpha_{z}\right\} \geq 0$ and $\Im\left\{\alpha_{z}\right\} \geq 0$.

Writing the continuity of $\phi$ and its normal derivative $\partial_{z} \phi$ in the interface plane $z=0$ leads to the following set of equations for the coefficients $A_{n}$ :

$$
\begin{aligned}
& \forall(m, n) \in \mathbb{N}^{2}, \\
& A_{m} \cos \left(\beta_{m} d\right)=\mathrm{j} \sum_{n \in \mathbb{N}} \Pi_{m n}(\alpha) \beta_{n} A_{n} \sin \left(\beta_{n} d\right),
\end{aligned}
$$

with $\Pi$ a matrix, function of $\alpha$ and the aspect ratio $d / L$ (see details in Appendix A).

Finally, Eq. (9) can be written in the matricial form

$$
D \overrightarrow{\mathcal{A}}=\overrightarrow{0}
$$

where $\mathcal{A}_{n}=A_{n} \sin \left(\beta_{n} d\right)$ and

$$
D_{m n}(\alpha)=\cot \left(\beta_{m} d\right) \delta_{m n}-\mathrm{j} \Pi_{m n} \beta_{n} .
$$

Then, the eigenvalues of the transverse eigenproblem are the values $\alpha_{i}$ of $\alpha$ for which $\operatorname{det}(D)=0$, and the eigenfunctions $\phi_{i}$ are given by the corresponding set of coefficients $A_{n}^{(i)}$, satisfying Eq. (10) with $D=D\left(\alpha_{i}\right)$. 


\section{B. Numerical resolution}

After truncation of Eq. (10) at a finite-size matricial problem, zeros of the determinant of $D$ are numerically located in the complex $\alpha$-plane to compute eigenvalues $\alpha_{i}$ of the transverse problem (Fig. 3).

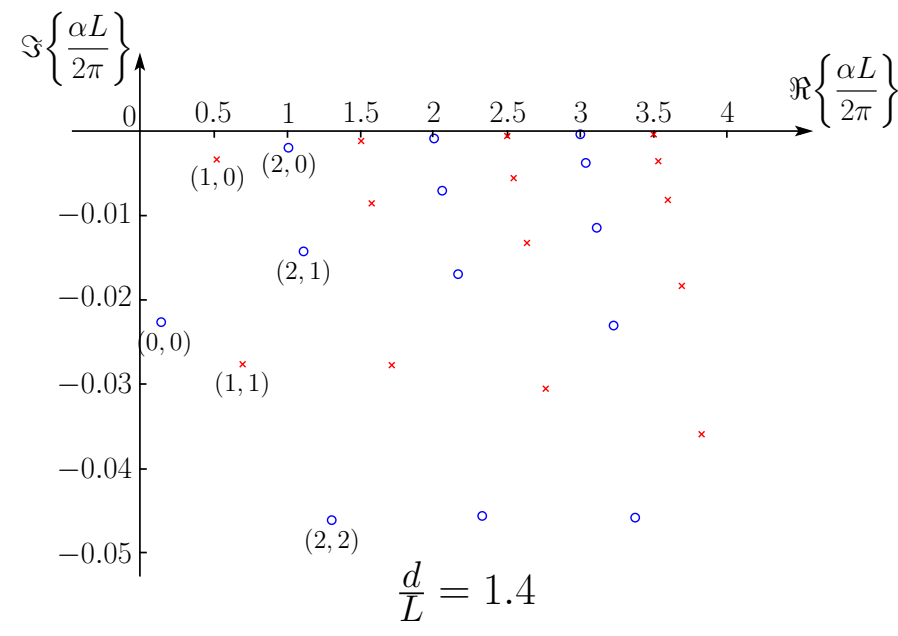

(a)

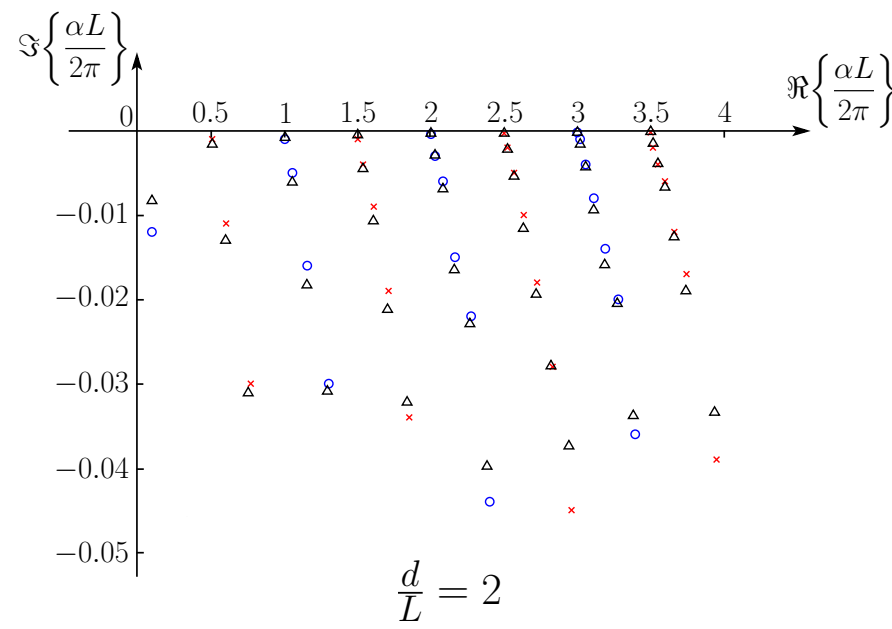

(b)

Figure 3. Spectrums of complex eigenvalues of the transverse problem for two different values of the aspect ratio: (a) $d / L=$ 1.4 , (b) $d / L=2$. '०': symmetric modes, ' $\times$ ': antisymmetric modes, ' $\triangle$ ': eigenvalues computed with FEM (color online).

Owing to the radiation losses in the infinite space $\Omega_{2}$ (above the waveguide), eigenvalues are complex, lying in the lower half-plane. ? ? ? The spectrum displays families of eigenvalues corresponding to either symmetric (blue circles 'o') or antisymmetric modes (red crosses ' $x$ '). An analogy with the classical, real, modes of the simple problem with a Dirichlet condition $(\phi=0)$ at $z=0$ instead of the exact radiating condition used in Sec. II.A, allows us to label the complex modes $\phi_{i}-$ at least the modes with eigenvalue located close enough to the real axis in the complex $\alpha$-plane - with a couple of integers denoting the number of vertical and horizontal nodal lines (Fig. 4). Following this terminology, the fam- ilies displayed in Fig. 3 are the $\phi_{(p, q)}$ with $p$ constant.

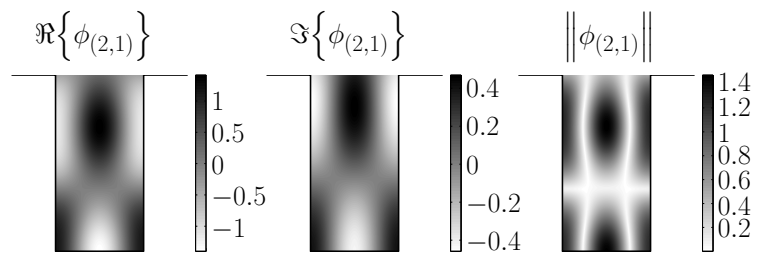

Figure 4. Symmetric mode $\phi_{(2,1)}$ (real part, imaginary part and modulus). The indices $(2,1)$ are chosen following an analogy with the classical eigenmodes in a closed cavity with a Dirichlet condition $\phi=0$ on the upper boundary: these indices denote the number of vertical and horizontal nodal lines.

Fig. 3 shows the spectrum of eigenvalues for two differents values of the aspect ratio of the cavity: (a) $d / L=1.4$, and (b) $d / L=2$. The pattern in both plots is similar, exhibiting the families of modes $\phi_{(p, q)}$ with $p$ constant. However, in the "deeper" cavity, with the aspect ratio $d / L=2$, the confinement of the modes is more important, thus, eigenvalues $\alpha_{i}$ have a smaller imaginary part than in the cavity with aspect ratio 1.4.

For comparison, a finite element method is used to solve the transverse eigenproblem (3). The semiinfinite space above the cavity is bounded with Perfectly Matched Layers (PML), as used by Koch? in a similar problem (Fig. 5).

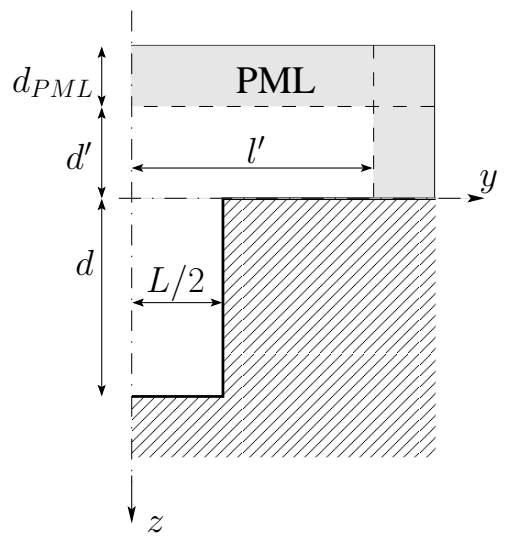

Figure 5. Geometry of the domain with PML in the FEM computations.

The results shown in this paper (Figs. 3-b and 6) have been obtained with parameters $d^{\prime}=L / 2, l^{\prime}=L$, $d_{\mathrm{PML}}=L / 2$, and $\tau_{y}=\tau_{z}=1+\mathrm{j}($ see Appendix B $) . \mathrm{A}$ Dirichlet condition $\phi=0$ is imposed on the outer boundaries of the PML. Moreover, as the geometry of the crosssection is symmetric about the $z$ axis, only one half of the domain is meshed, with the approriate symmetry or antisymmetry condition imposed at $y=0$. Computations have been performed using The Partial Differential Equation toolbox from Matlab ${ }^{\circledR}$.

The results of the two compared methods - the resolution of Eq. (10) and the FE Method - are in good 


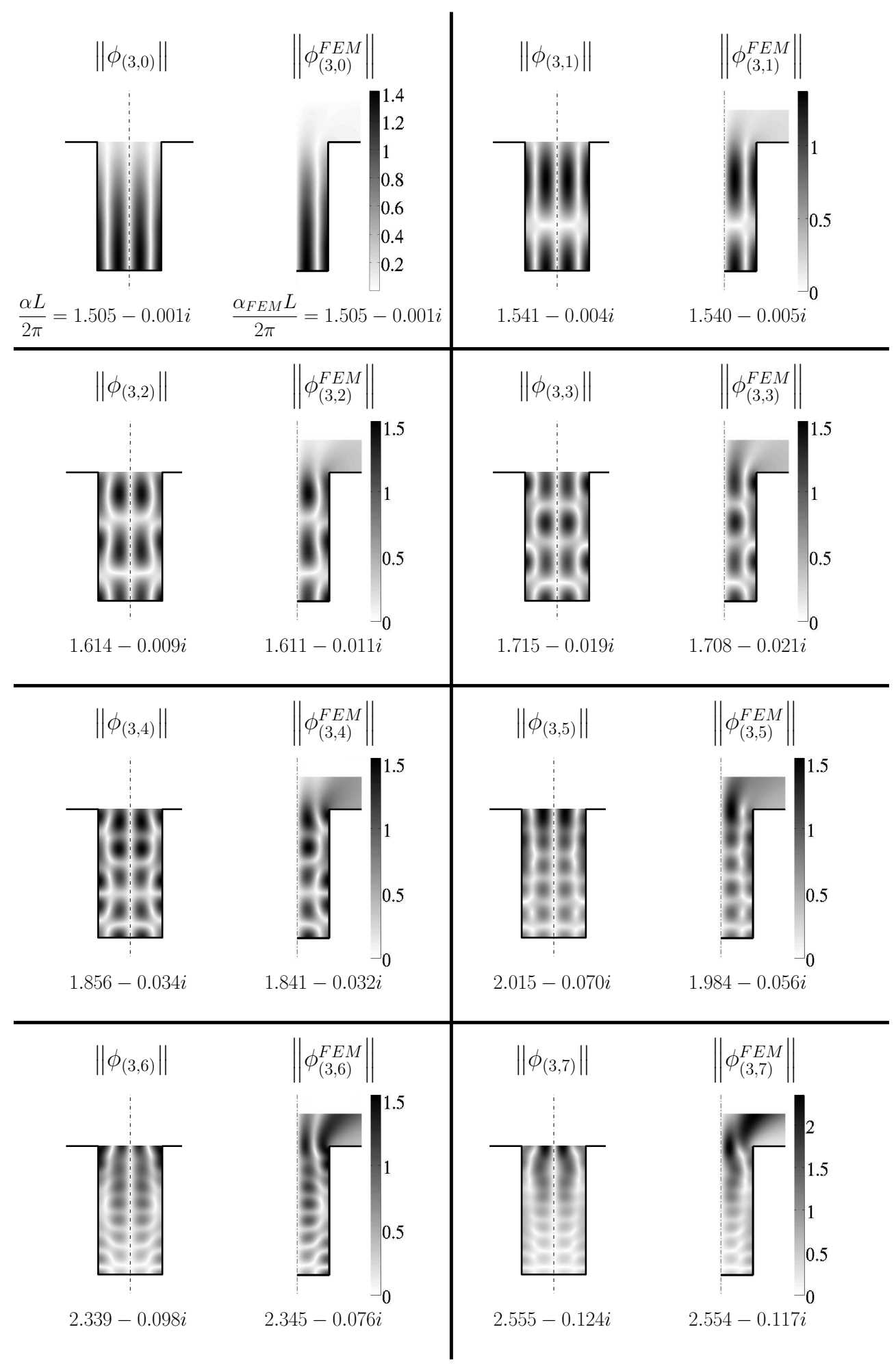

Figure 6. Antisymmetric eigenfunctions $\phi_{(3, q)}, q>0$, and comparison with FEM computations. The PML domain is not shown in the FEM results.

agreement (Fig. 3-b). The discrepancy between the results increases for larger values of the imaginary part $\Im\left\{\alpha_{i}\right\}$. However, eigenvalues that are less well-estimated are associated to modes that will be strongly attenuated when propagated in the waveguide. They are, then of secondary importance when considering the transport of energy on a sufficiently long distance. Moreover, it will be shown in the following that the contribution of these modes in the determination of the sets of coefficients $\left\{a_{i}\right\}$ and $\left\{b_{i}\right\}$ is almost negligible.

A comparison between the eigenfunctions $\phi_{i}$ deduced from the method detailed in $\S$ II.A and from the FEM 
computation shows also a good agreement (Fig. 6). Both methods give very similar results, even when the error on the imaginary part of $\alpha_{i}$ becomes more significant (Fig. 6, bottom). The figure also clearly shows that low order modes weakly "radiate" in the infinite space $\Omega_{2}$ : the effect of the opening of the waveguide appears as a small perturbation on the classical, non radiating, solutions that would be obtained by applying an homogeneous Dirichlet condition at the top of the waveguide $(z=0)$. For higher order modes, however, eigenfunctions $\phi_{i}$ differ more and more from the "Dirichlet" solutions. Patterns of nodal lines are more complex - consequently, the indexation with indices $(p, q)$ become less relevant - and the confinement, that was strong for low order modes, becomes weaker, with the energy increasing near the interface $z=0$.

Now that the transverse eigenmodes are determined, they can be used to perform a multimodal formulation of the sound propagation within a street canyon.

\section{PROPAGATION ALONG THE STREET}

As explained in Sec. I, the transverse modes $\phi_{i}$ can be used, for given source and radiation conditions at the ends of the waveguide, to built a solution of the wave equation, as written in Eq. (4).

Because the transverse eigenvalues $\alpha_{i}$ are complex with $\Im\left\{\alpha_{i}\right\}<0$, the propagation constants $k_{i}$ are also complex, with $\Im\left\{k_{i}\right\}>0$, even for real source frequency $\omega$. Then, all the modes $\phi_{i} \exp \left( \pm \mathrm{j} k_{i} x\right)$ in the waveguide decrease exponentially while propagating, reflecting the radiation losses during the propagation along the open waveguide. This corresponds to leaky modes.

The two sets of coefficients $\left\{a_{i}\right\}$ and $\left\{b_{i}\right\}$ must be found, as functions of the end conditions in the waveguide. At the input end of the waveguide, a source condition is defined as a given acoustic pressure distribution in a plane $x=$ constant, with frequency $\omega$. For example, at $x=0: p(0, y, z, \omega)=p_{0}(y, z) \exp (\mathrm{j} \omega t)$. At the output end of the waveguide is given a radiation condition.

\section{A. Input condition}

Let call $P_{i}(x)=a_{i} \exp \left(\mathrm{j} k_{i} x\right)+b_{i} \exp \left(-\mathrm{j} k_{i} x\right)$ the coefficients in the development in series (4). Since the modes $\phi_{i}$ are not orthogonal, the initial field $p_{0}(y, z, \omega)$ cannot be projected on the $\left\{\phi_{i}\right\}$ as it is classicaly made to find the $\left\{P_{i}(0)\right\}$. Thus, after truncation at a finite number $\mathcal{N}$ of terms in the development (4), a least square method is thus used to find these coefficients ${ }^{\text {? }}$ :

$$
\vec{P}(0)=\left(\Lambda^{(\phi)}\right)^{-1} \vec{p}_{0}^{(\phi)}
$$

where the $i$-th component of $\vec{P}(0)$ is $P_{i}(0)$ and

$$
\begin{gathered}
\Lambda_{i j}^{(\phi)}=<\phi_{i} \mid \phi_{j}>, \\
p_{0 i}^{(\phi)}=<\phi_{i} \mid p_{0}>,
\end{gathered}
$$

with the product

$$
<f \mid g>=\iint \bar{f} g \mathrm{~d} \Omega .
$$

Practically, we consider source conditions $p_{0}(y, z, \omega)$ with a support included in $\Omega_{1}$, i.e., inside the street canyon. Thus, for convenience when determining $\vec{P}(0)$, we consider the restriction of the eigenfunctions $\phi_{i}$ to the domain $\Omega_{1}$, that is,

$$
\begin{aligned}
& \forall(y, z) \in\left[-\frac{L}{2}, \frac{L}{2}\right] \times[0, d] \\
& \phi_{i}(y, z)=\sum_{n \in \mathbb{N}} A_{n}^{(i)} \cos \left(\beta_{n}^{(i)}(d-z)\right) \psi_{n}(y),
\end{aligned}
$$

and the product $<\mid>$ above is defined as

$$
<f \mid g>=\int_{0}^{d} \int_{-L / 2}^{L / 2} \bar{f} g \mathrm{~d} y \mathrm{~d} z .
$$

Note that in the case of real orthogonal modes, as in classical closed waveguides, the least square method gives the usual projection coefficients $P_{m}=<\phi_{m} \mid p>$.

\section{B. Output condition}

Let $Q_{i}(x)=\mathrm{j} k_{i}\left(a_{i} \exp \left(\mathrm{j} k_{i} x\right)-b_{i} \exp \left(-\mathrm{j} k_{i} x\right)\right)$ be the coefficients in the development of the $x$-component of $\vec{\nabla} p$. One assumes that at the output end of the waveguide, say, at $x=x_{\text {end }}$, the condition is given as an admittance matrix $Y_{\text {end }}$ fulfilling $\vec{Q}\left(x_{\text {end }}\right)=Y_{\text {end }} \vec{P}\left(x_{\text {end }}\right)$.

Again, as for the formulation at the input end, and due to the non-orthogonality of the eigenmodes, the matrix $Y_{\text {end }}$, for some complex end conditions, may not be straightforwardly calculated. However, usual end conditions - rigid end, non radiating open end, anechoic termination - can be easily formulated with this type of admittance matrix, generalization for all modes of the usual admittance for the plane wave? . For the results we will consider anechoic termination, i.e. $b_{i}=0$.

\section{Solutions for $\left\{a_{i}\right\}$ and $\left\{b_{i}\right\}$}

Now that an input condition $\vec{P}(O)$ and an output condition $Y_{\text {end }}$ are known, the vectors $\vec{a}$ and $\vec{b}$ of the $\left\{a_{i}\right\}$ and $\left\{b_{i}\right\}$ in Eq. (4) can be calculated ${ }^{?}$ ? :

$$
\begin{aligned}
& \vec{a}=(1-\delta)^{-1} \vec{P}(0), \\
& \vec{b}=-\delta(1-\delta)^{-1} \vec{P}(0),
\end{aligned}
$$

where $\delta=D_{1}\left(Y_{\text {end }}+Y_{\mathrm{c}}\right)^{-1}\left(Y_{\text {end }}-Y_{\mathrm{c}}\right) D_{1}, D_{1}$ is diagonal with terms $D_{1 i}=\exp \left(\mathrm{j} k_{i} x_{\text {end }}\right), Y_{\mathrm{c}}$ is diagonal with terms $Y_{\mathrm{cn}}=\mathrm{j} k_{i}$. Thus, with these solutions for $\vec{a}$ and $\vec{b}$, the pressure field in the open waveguide can be calculated. 
However, terms $\exp \left(-\mathrm{j} k_{i} x\right)$ can be the source of numerical problems of convergence. Then, defining $\overrightarrow{\vec{b}}=D_{1}^{-1} \vec{b}$, the pressure field is written

$$
p^{(N)}(x, y, z, \omega)=\sum_{i=1}^{N}\left(a_{i} e^{\mathrm{j} k_{i} x}+\tilde{b}_{i} e^{\mathrm{j} k_{i}\left(x_{\mathrm{end}}-x\right)}\right) \phi_{i}(y, z)
$$

with $N \leq \mathcal{N}$. This new formulation depends only on $D_{1}$, not on $D_{1}^{-1}$, and on exponentials with positive arguments $x$ or $L-x$.

\section{RESULTS}

In the following, for simplicity, we will consider the wave field downstream from a source in an infinite waveguide. Then, there are no back propagated waves: $b_{i}=0$.

\section{A. Input condition}

The initial condition at $x=0$ is the pressure distribution shown in Fig.7 (left part) and given by

$$
p_{0}(y, z)=\sum_{k=1}^{3} \frac{1}{\sigma_{k} \sqrt{2 \pi}} e^{\frac{-\left(y-y_{k}\right)^{2}-\left(z-z_{k}\right)^{2}}{2 \sigma_{k}^{2}}}
$$

where $\sigma_{k} \in \mathbb{R}^{+}$and $\left(y_{k}, z_{k}\right) \in[-L / 2, L / 2] \times[0, d]$. The associated dimensionless frequency is $k L / 2 \pi=1.2$. This input condition is chosen as a non trivial solution for the modal formulation.

Then, the $\left\{a_{i}\right\}$ are found by substituting Eq. (20) for $p_{0}(y, z)$ in Eqs. (12-13). The modal reconstruction is shown in Fig. 7. Using a basis of $N=\mathcal{N}=30$ modes (we recall that $N$ is the number of terms in the series (19) and $\mathcal{N}$ is the size of the linear problem (12) in the least square estimation of the $\left\{a_{i}\right\}$, so that $\left.N \leq \mathcal{N}\right)$, the input pressure condition is well reproduced with a residual error of $3.6 \%$. This error is due to the high order depth modes of the first families that have deliberately not been considered in the modal basis because of their weak of relevance in the propagation. Furthermore, it will be shown in the following that omitting these modes does not affect significantly the estimation of the $\left\{a_{i}\right\}$ for the modes taken into account.

To evaluate the convergence of the method when increasing the number $N$ of modes taken into account in Eq. (19), from a $\mathcal{N}=30$ modes basis, an error $\epsilon$ is defined as

$$
\epsilon=\sqrt{\frac{\int_{0}^{d} \int_{-\frac{L}{2}}^{\frac{L}{2}}\left\|p^{(N)}-p_{0}\right\|^{2} \mathrm{~d} y \mathrm{~d} z}{\int_{0}^{d} \int_{-\frac{L}{2}}^{\frac{L}{2}}\left\|p_{0}\right\|^{2} \mathrm{~d} y \mathrm{~d} z}},
$$

where $p^{(N)}$ is the modal solution obtained with $N$ modes (Eq. 19), and $p_{0}$ the reference field. The modes are sorted by increasing value of the imaginary part of their propagation constant $k_{i}$, that is, from the least damped to the most damped leaky mode propagating along the canyon
Input source condition
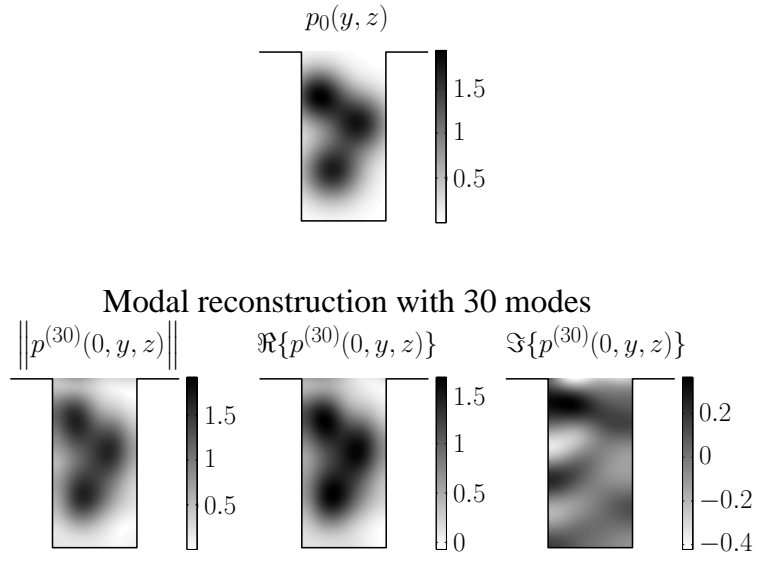

Figure 7. Top : Initial condition at abscissa $x=$ 0 ; The parameters of the three Gaussian functions in Eq. (20) are $\left(y_{1}, z_{1}, \sigma_{1}\right)=(0.18 L, 0.42 d, 0.24),\left(y_{2}, z_{2}, \sigma_{2}\right)=$ $(-0.30 L, 0.26 d, 0.22), \quad\left(y_{3}, z_{3}, \sigma_{3}\right)=(-0.14 L, 0.70 d, 0.24)$. Bottom: Modal reconstruction of the initial condition $p_{0}(y, z)$, using $N=\mathcal{N}=30$ modes.

Table I. Classification of the modes by increasing value of $\Im\left\{k_{i}\right\}$ at frequency $k L / 2 \pi=1.2$.

\begin{tabular}{cccc}
\hline \hline Classification & Couple $(m, n)$ & Classification & Couple $(m, n)$ \\
\hline $1^{\text {st }}$ & $(1,0)$ & $6^{\text {th }}$ & $(0,1)$ \\
$2^{\text {nd }}$ & $(0,0)$ & $7^{\text {th }}$ & $(1,2)$ \\
$3^{\text {rd }}$ & $(2,0)$ & $8^{\text {th }}$ & $(2,2)$ \\
$4^{\text {th }}$ & $(1,1)$ & $9^{\text {th }}$ & $(0,2)$ \\
$5^{\text {th }}$ & $(2,1)$ & $10^{\text {th }}$ & $(1,3)$ \\
\hline \hline
\end{tabular}

(Tab. I). This type of classification depends directly on each eigenvalue and the source frequency.

The convergence of the reconstruction of the initial condition $p_{0}(y, z)$ is shown in Fig. 8 (circles 'o'). Naturally in such a modal approach, depending on the source distribution, each mode introduced in the computation contributes differently to the reconstruction of $p_{0}(y, z)$.

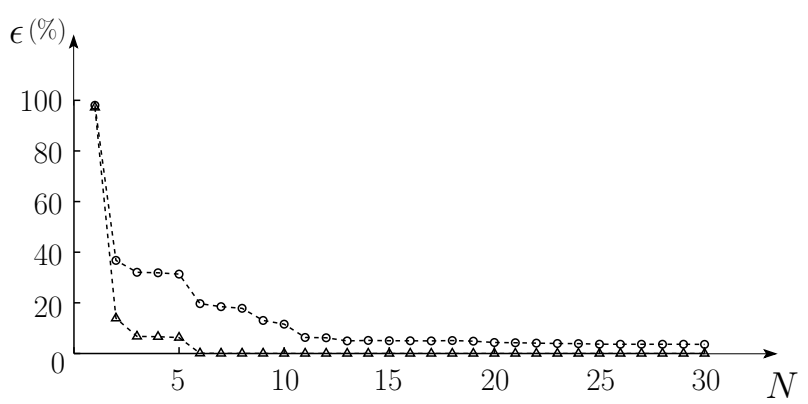

Figure 8. Evolution of the error indicator $\epsilon$ with the the number of modes $N$ taken into account in the computation of the solution. 'o': the error is computed at $x=0$, with $\epsilon$ given in Eq. (21). ' $\triangle$ ': the error is computed at $x=10 L$, with $p^{(30)}(10 L, y, z, \omega)$ as the reference field. 


\section{B. Propagation within the waveguide}

Assuming that the convergence is reached for $N=$ 30 , the field $p^{(30)}(x, y, z, \omega)$ is now taken as the reference field to compute an estimation error at abscissa $x=10 \mathrm{~L}$ (triangles ' $\triangle$ ' in Fig. 8). The variability, depending on the initial condition $p_{0}(y, z)$, of the contributions of the modes to the congergence is still visible, but, moreover, it clearly appears that only a few modes - the less damped modes - still contribue to the transport of energy at that distance from the "source". Practically, sorting the modes as done in Tab. I is thus a good choice to increase the convergence, as soon as one is interested by the wave field in the street canyon at a sufficient distance from the source.

Fig. 9 shows the field in the cross-section of the waveguide at abscissa $x=10 L, L$ the width of the waveguide. The left plot is obtained using Eq. (19) and $N=30$ - the total number of modes used to perform the least square estimation -, while the right plot is obtained using only the first six modes, with the ordering defined above. Both results are very close: the relative error between them, defined as in Eq. (21), is less than $0.35 \%$.
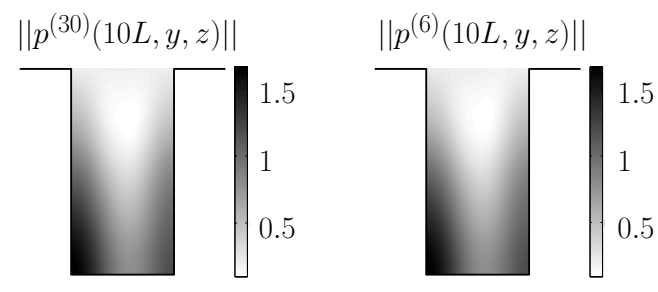

Figure 9. Modal field at $x=10 L$ using $N=30$ modes (left) and $N=6$ modes (right).

Since only a few modes are necessary to describe the field at certain distance from the source, it would be advantageous to use a reduced modal basis in the computation. Since modes are not orthogonal, the value of each modal coefficient $a_{i}$ depends on the size of the "basis" used in the least square estimation. One shows, however, that this dependency is rapidly weak, in particular for the first modes, that is, the less damped (Fig. 10). The notation $a_{i}^{(\mathcal{N})}$ is used to denote the number of modes $\mathcal{N}$ taken into account for the least square estimation of $a_{i}$.

To evaluate the relevance of using a reduced modal basis, two modal solutions of the wave equation in the infinite street canyon with the initial condition (20) at $x=0$ are compared: the solution with $N=\mathcal{N}=10$, and the solution with $N=\mathcal{N}=30$. The relative error between these two solutions, as defined in Eq. (21), is plotted in Fig. 11 as function of the distance from the source plane.

Naturally, the source condition $p_{0}(y, z)$ is badly reconstructed with a limited number of modes, and the relative error near the plane $x=0$ is thus significant. But the error decreases rapidly, to be under $1 \%$ after less than two widths. It follows that the acoustic field, rapidly, is "carried" by a small number of modes, the less damped

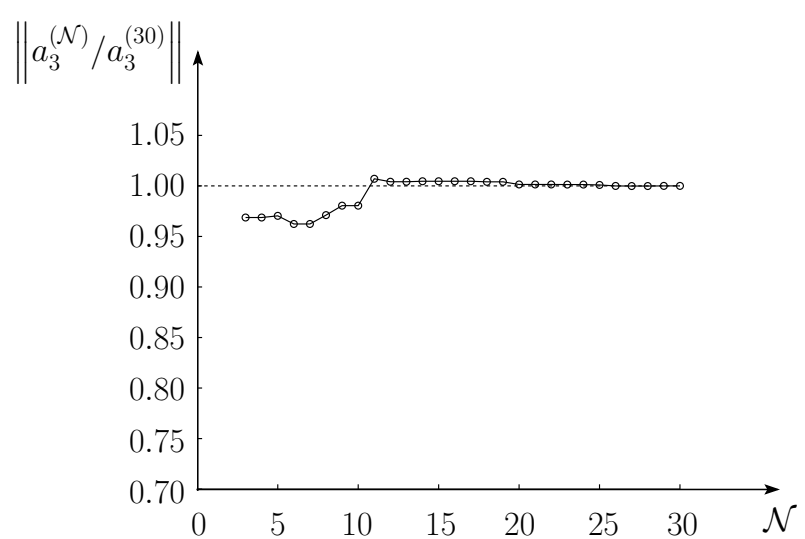

(a)

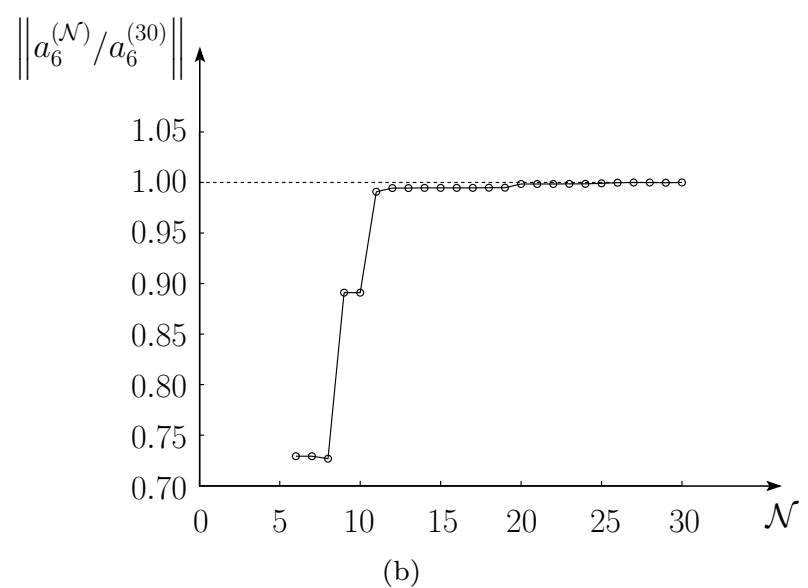

Figure 10. Evolution of the modal coefficients $a_{3}$ (a) and $a_{6}$ (b) with the number of modes $\mathcal{N}$ used for the least square estimation.

modes, that are therefore well confined and guided in the open geometry of the street canyon. This points out a double interest of the modal formulation using leaky modes in a street canyon; first, as a physically relevant approach, describing the competitive effects of confinement and radiation in such an open geometry; second, as an efficient numerical method, since a few modes only is used to accurately model the wave propagation in the waveguide.

\section{CONCLUSIONS}

The problem of sound propagation in a urban street canyon is solved using a multimodal formulation that gives the acoustic field as a sum on the leaky modes in the canyon. The leaky modes, that naturally reflects the competitive effects of confinement and radiation of the wave in such partially bounded geometries, can be numerically determined. As these are complex modes that decay exponentially while propagating, the number of modes that effectively carry the wave field (emitted by some source in the waveguide) decreases rapidly, so that only a few modes, at a sufficiently large distance from the source, is necessary to accurately model the 


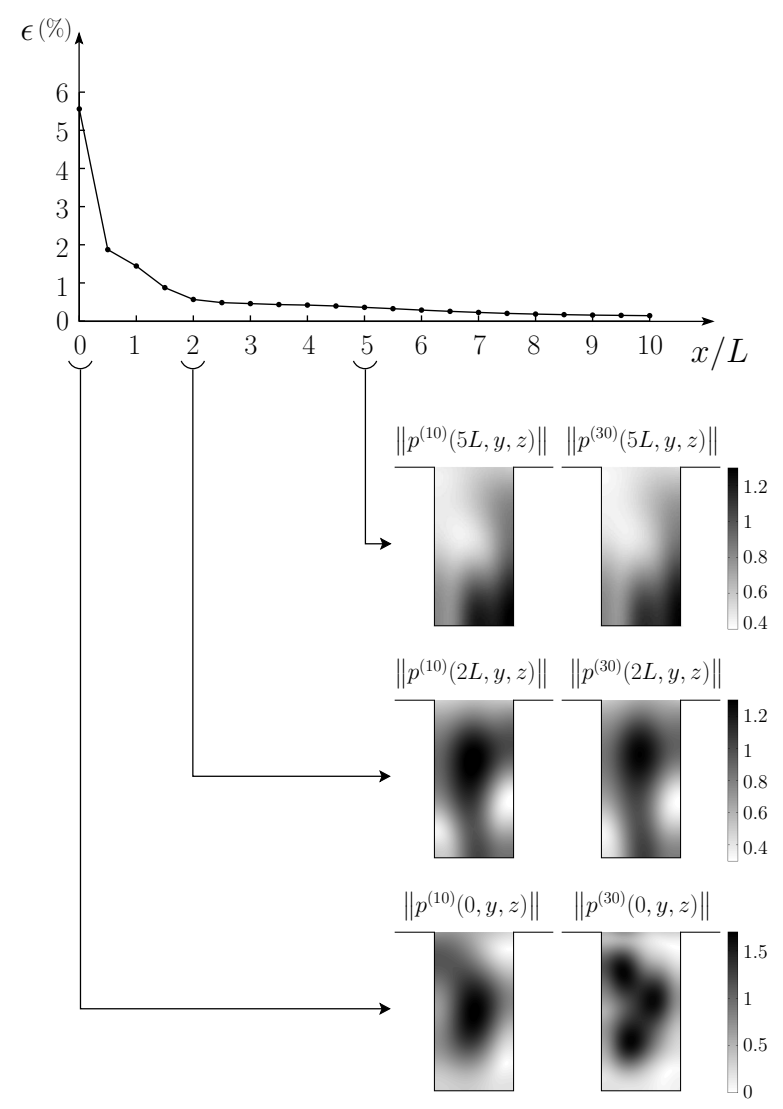

Figure 11. Relative error (as defined by Eq. 21) between the modal solution (19) with $N=\mathcal{N}=10$ and the same modal solution with $N=\mathcal{N}=30$, as function of the distance from the source plane.

wave propagation. This gives this approach a real interest for numerical computations, in addition to its interest as a physically meaningfull description of the street as a partially confining and guiding medium for the acoustic waves.

\section{Appendix A: ESTABLISHEMENT OF EQ. (9)}

Continuity equations in the interface plane $z=0,|y|<$ $L / 2$ are

$$
\phi\left(y, z=0^{+}\right)=\phi\left(y, z=0^{-}\right)
$$

and

$$
\frac{\partial \phi\left(y, z=0^{+}\right)}{\partial z}=\frac{\partial \phi\left(y, z=0^{-}\right)}{\partial z} .
$$

A first relation between the $\left\{A_{n}\right\}$ and $B$ is found by substituting Eqs. (6) and (8) for $\phi$ in combined Eqs. (5a) and (A2):

$$
\sum_{n \in \mathbb{N}} A_{n} \beta_{n} \sin \left(\beta_{n} d\right) \psi_{n}(y)=-\mathrm{j} \frac{1}{2 \pi} \int_{-\infty}^{+\infty} B \alpha_{z} e^{\mathrm{j} \alpha_{y} y} \mathrm{~d} \alpha_{y}
$$

to find

$$
B=\mathrm{j} \sum_{n \in \mathbb{N}} A_{n} \beta_{n} \sin \left(\beta_{n} d\right) \frac{S_{n}\left(\alpha_{y}\right)}{\alpha_{z}}
$$

where

$$
S_{n}\left(\alpha_{y}\right)=\int_{-L / 2}^{L / 2} \psi_{n}(y) e^{-\mathrm{j} \alpha_{y} y} \mathrm{~d} y
$$

A second relation is found by using the continuity equation (A1):

$$
\sum_{n \in \mathbb{N}} A_{n} \cos \left(\beta_{n} d\right) \psi_{n}(y)=\frac{1}{2 \pi} \int_{-\infty}^{+\infty} B e^{\mathrm{j} \alpha_{y} y} \mathrm{~d} \alpha_{y}
$$

whence it follows that

$$
A_{m} \cos \left(\beta_{m} d\right)=\frac{1}{2 \pi} \int_{-\infty}^{+\infty} B S_{m}^{*}\left(\alpha_{y}\right) \mathrm{d} \alpha_{y}
$$

Then, Eqs. (A4) and (A7) leads to the set of linear, homogenous equations (9) for the $\left\{A_{n}\right\}: \forall m \in \mathbb{N}$,

$$
A_{m} \cos \left(\beta_{m} d\right)=\mathrm{j} \sum_{n \in \mathbb{N}} \Pi_{m n}(\alpha) \beta_{n} A_{n} \sin \left(\beta_{n} d\right)
$$

where

$$
\Pi_{m n}=\frac{1}{2 \pi} \int_{-\infty}^{+\infty} \frac{S_{m}^{*} S_{n}}{\alpha_{z}} \mathrm{~d} \alpha_{y}
$$

It is easily shown that, for real values of $\alpha_{y}, \Pi_{m n}$ vanishes unless $m$ and $n$ have the same parity. $T$ Then, Eq. (9) breaks up into two sets of linear equations, one governing the symmetrical eigenmodes (even functions of $y$ with even values of $m$ and $n$ ), the other governing antisymmetrical eigenmodes (odd functions of $y$ with odd values of $m$ and $n$ ). The following equation gives a general expression of $\Pi_{m n}$ for both even or odd indices $m$ and $n$ : 


$$
\Pi_{m n}(\alpha)=(-1)^{\frac{3 m+n}{2}} \frac{\sqrt{2-\delta_{m 0}} \sqrt{2-\delta_{n 0}}}{\pi} \int_{0}^{+\infty} \frac{\alpha_{y}^{2}}{\alpha_{z}\left(\alpha_{y}+\frac{m \pi}{L}\right)\left(\alpha_{y}+\frac{n \pi}{L}\right)} \operatorname{sinc}\left(\frac{\alpha_{y}-\frac{m \pi}{L}}{2}\right) \operatorname{sinc}\left(\frac{\alpha_{y}-\frac{n \pi}{L}}{2}\right) \mathrm{d} \alpha_{y} .
$$

\section{Appendix B: FORMULATION IN THE PERFECTLY MATCHED LAYERS}

PML are used as a to avoid non-physical reflections at the boundaries of a necessarily finite domain in a numerical computation. The method works as follows: the solution $\phi(y, z)$ of the eigenproblem (3) above the cavity is analytically continued in the PML with respect to variables $(y, z)$ to complex variables $(\hat{y}, \hat{z})$. The extended solution $\hat{\phi}$ satisfies

$$
\left(\frac{\partial^{2}}{\partial \hat{y}^{2}}+\frac{\partial^{2}}{\partial \hat{z}^{2}}\right) \hat{\phi}=0
$$

Complex variables $(\hat{y}, \hat{z})$ are now written

$$
\hat{y}(y)=\int_{0}^{y} \tau_{y}\left(y^{\prime}\right) \mathrm{d} y^{\prime}, \quad \hat{z}(z)=\int_{0}^{z} \tau_{z}\left(z^{\prime}\right) \mathrm{d} z^{\prime}
$$

with $\Re\left\{\tau_{y, z}\right\}>0, \Im\left\{\tau_{y, z}\right\}>0$, and $\tau_{y}\left(y \leq l^{\prime}\right)=1$, $\tau_{z}\left(z \leq d^{\prime}\right)=1$ (Fig. 5). The results in this paper have been obtained with $\tau_{y}\left(y>l^{\prime}\right)=\tau_{z}\left(z>d^{\prime}\right)=1+\mathrm{j}$. 